\title{
First-line an liposomales Doxorubicin denken
}

\begin{abstract}
Die First-line-Behandlung mit nicht-pegyliertem liposomalem Doxorubicin ist für Patientinnen mit metastasiertem Mammakarzinom und adjuvanter Anthrazyklin/Taxan-haltiger Vorbehandlung eine Therapieoption, die noch einmal ein gutes Ansprechen bei guter Verträglichkeit induzieren kann.
\end{abstract}

Patientinnen mit metastasiertem Mammakarzinom sind oft bereits adjuvant mit einer Anthrazyklin/Taxan-haltigen Chemotherapie behandelt. Bei langem therapiefreiem Intervall ist für die First-line-Behandlung die Re-Induktion eine vielversprechende Therapieoption, erläuterte Sherko Kümmel, Essen. Die Re-Induktion benötige auch „Fingerspitzengefühl“. In der Regel ist nicht die Wirksamkeit der limitierende Faktor, sondern die Verträglichkeit, speziell die kumulative Toxizität, wie z.B. periphere Polyneuropathie bei Paclitaxel oder Kardiotoxizität bei den Anthrazyklinen. Letztere verliert deutlich an klinischer Relevanz, wenn die Re-Induktion mit nicht-pegyliertem liposomalem Doxorubicin durchgeführt wird, so Kümmel.

\section{Gute Wirksamkeit und Verträglichkeit}

Die Substanz habe den Vorteil, in der First-line-Situation bei Anthrazyklin-vorbehandelten Patientinnen noch einmal „den vollen Effekt bei guter Verträglichkeit" zu erreichen, betonte Kümmel. Klinische Studienergebnisse bei Anthrazyklin-vorbehandelten Patientinnen zeigen bei der Behandlung mit nicht-pegyliertem liposomalem Doxorubicin eine objektive Ansprechrate (komplette plus partielle Remission [CR/PR]) von 50\%, die damit mehr als doppelt so hoch lag wie unter konventionellem Doxorubicin (20\%; Batist G et al., 2001, J Clin Oncol 19: 1444-1454).

Zudem hat liposomales Doxorubicin eine gute kardiale Verträglichkeit. Durch die liposomale Verkapselung des Wirkstoffs wird dieser wesentlich gezielter zur Tumorzelle transportiert. Andere Organsysteme werden deutlich weniger belastet. Die kumulative Gesamtdosis, ab der ein erhöhtes kardiales Risiko besteht, liegt bei liposomalem Doxorubicin etwa um den Faktor fünf höher als bei konventioneller Gabe des Anthrazyklins. Die Gefahr, die kumulative Gesamtdosis zu überschreiten, ist damit laut Kümmel deutlich reduziert. Kümmel wies außerdem darauf hin, dass die Wirksamkeit des nicht-pegylierten liposomalen Doxorubicins unabhängig vom HER2 (Human Epidermal Growth Factor Receptor 2)-Status ist. $B K P$

Quelle: Veranstaltung der Cephalon $\mathrm{GmbH}$

First-line-Therapie bei follikulärem Non-Hodgkin-Lymphom

\section{Erhaltungstherapie mit Rituximab reduziert das Progressionsrisiko}

Das zeigen die Ergebnisse der Phase-III-Studie PRIMA (Primary Rituximab and Maintenance) zur First-line-Therapie bei Patienten mit follikulärem Non-Hodgkin-Lymphom.

In der Studie wurden Patienten mit follikulärem Lymphom, die auf eine First-line-Immunochemotherapie mit Rituximab angesprochen hatten, randomisiert entweder für zwei Jahre mit Rituximab $\left(375 \mathrm{mg} / \mathrm{m}^{2}\right.$ KOF alle acht Wochen; $\left.n=505\right)$ weiterbehandelt oder für zwei Jahre beobachtet $(n=513)$. Primärer Endpunkt war das progressionsfreie Überleben seit der Randomisierung.

Bereits in der vorab geplanten Zwischenanalyse nach median 25 Monaten Follow-up wurde der primäre Endpunkt erreicht: Es zeigte sich ein signifikanter Vorteil im progressionsfreien Überleben zugunsten der Rituximab-Erhaltungstherapie (Abb.). Ein Nutzen der Rituximab-Erhaltungstherapie wurde dabei unabhängig vom Alter ( $<60$ Jahre, $\geq 60$ Jahre), FLIPI (Follicular Lymphoma International Prognostic Index) $(\leq 1,2, \geq 3)$ und Ansprechen auf die Induktionstherapie (partielles bzw. komplettes Ansprechen) beobachtet. Neue oder unerwartete Toxizitäten wurden bei der Erhaltungstherapie mit Rituximab in der First-line-Situation nicht festgestellt. Der Nutzen der Ri-

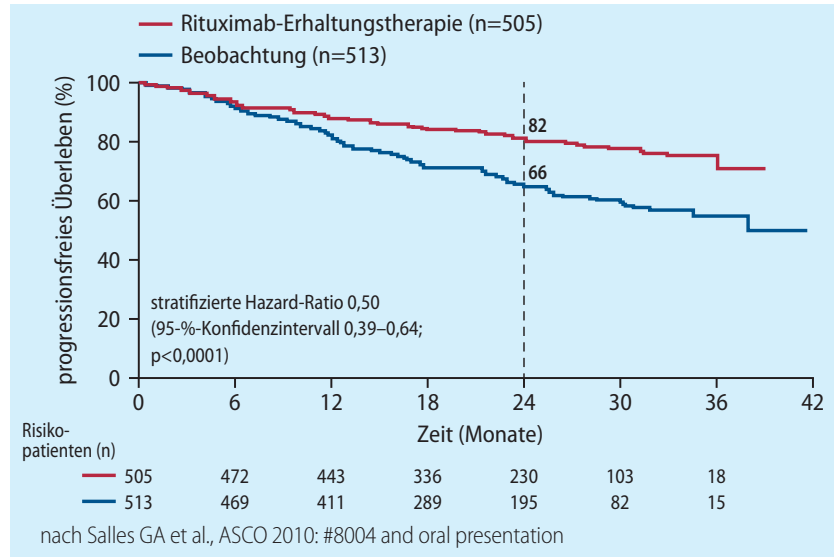

PRIMA-Studie - progressionsfreies Überleben (primärer Endpunkt) seit der Randomisierung: Rituximab-Erhaltungstherapie versus Beobachtung

tuximab-Erhaltungstherapie ist laut Gilles A. Salles, Lyon/Frankreich, in der First-line-Situation größer als bei Patienten mit rezidivierter Erkrankung. Den Studienergebnissen zufolge sollte die RituximabErhaltungstherapie neuer Behandlungsstandard für Patienten mit nicht vorbehandeltem follikulärem Lymphom sein. $A M$ 